\title{
Hepatic abnormalities in coeliac disease: three cases of delayed diagnosis
}

\author{
H.C. Mitchison ${ }^{1}$, C.O. Record ${ }^{2}$, M.C. Bateson ${ }^{3}$ and I. Cobden ${ }^{1}$ \\ ${ }^{1}$ Preston Hospital, North Tyneside, ${ }^{2}$ Royal Victoria Infirmary Newcastle upon Tyne, and ${ }^{3}$ Bishop Auckland \\ Hospital, County Durham, UK.
}

\begin{abstract}
Summary: Three cases of biopsy-proven coeliac disease are presented. In each case the predominant clinical and laboratory features suggested liver disease, bowel symptoms were a minor part of the presentation and the diagnosis of coeliac disease was not reached for approximately 6 months. Liver biopsy in one case showed marked fatty change, in the other cases only mild hepatitic changes. A gluten-free diet produced resolution of symptoms and, in the patient with steatosis, normalization of liver function tests. Hepatic abnormalities have been reported in coeliac disease and the significance of these is discussed but such abnormalities are usually minor and do not obscure the underlying diagnosis. We feel that these cases serve as a salutary reminder of the protean manifestations of coeliac disease.
\end{abstract}

\section{Introduction}

Coeliac disease is recognized as being associated with a variety of hepatic abnormalities, ${ }^{1-10}$ most of which are noted in passing and are of secondary clinical importance. We report 3 cases with coeliac disease where the abnormalities in liver function so dominated the clinical picture that there was considerable delay in recognizing the primary pathology.

\section{Case reports}

\section{Case 1}

A 40 year old Caucasian woman presented with a 2-month history of weight loss and right hypochondrial pain with, initially, diarrhoea and vomiting. The symptoms had started after a course of penicillin; the vomiting had settled spontaneously, the diarrhoea after treatment with ispaghula. There was no relevant past medical, family or drug history. She did not smoke and drank less than $80 \mathrm{~g}$ alcohol/week. On examination she had long-standing chloasma but no cutaneous stigmata of chronic liver disease. A smooth non-tender liver edge was palpable $4 \mathrm{~cm}$ below the costal margin. Sigmoidoscopy was normal.

Investigations revealed anaemia (haemoglobin

Correspondence: H.C. Mitchison, M.A., M.R.C.P., Level 6, Freeman Hospital, Newcastle upon Tyne NE7 7DN, UK. Accepted: 1 June 1989
$10.7 \mathrm{~g} / \mathrm{dl}$ ) with target cells, hypochromia and $\cong$ anisocytosis, and abnormal liver function tests $-\frac{8}{0} \overrightarrow{0}$ bilirubin $20 \mu \mathrm{mol} / 1$ (normal $\leqslant 17$ ), aspartate transaminase (AST) $130 \mathrm{IU} / 1$ (normal $\leqslant 32$ ), alkaline phosphatase (ALP) $289 \mathrm{IU} / 1$ (normal $\leqslant 90$ ) and albumin $28 \mathrm{~g} / \mathrm{l}$. Prothrombin time was prolonged, ratio 1.6:1. Serum iron was low with normal vitamin B12 and folate. Serum markers for hepatitis A and B were negative but IgG antinuclear factor was positive, titre 1/80. Gastroscopy and barium enema were normal. Abdominal ultrasound and isotope liver scan confirmed hepatomegaly but showed no focal defects or splenomegaly. A diagnosis of chronic active hepatitis was considered; however liver biopsy showed severe and widespread fatty change but no inflammatory cells (Figure 1).

The patient continued to deteriorate with persistent abdominal pain and weight loss, the return of diarrhoea, increasing hepatic enlargement and peripheral oedema. Liver function tests worsened with AST 202 IU/l. Computerized tomography, seeking intraabdominal neoplasia, showed only an enlarged liver with fatty infiltration. Small bowel barium followthrough revealed coarsening of the small bowel mucosa and prolonged transit. The diagnosis was eventually reached when a small bowel biopsy showed total villous atrophy, crypt hyperplasia with an increase in mitoses, and a moderate plasma cell and lymphocyte infiltrate. Institution of a gluten-free diet resulted in total resolution of her symptoms within 5 weeks. Within 3 months she had gained $10 \mathrm{~kg}$ in 


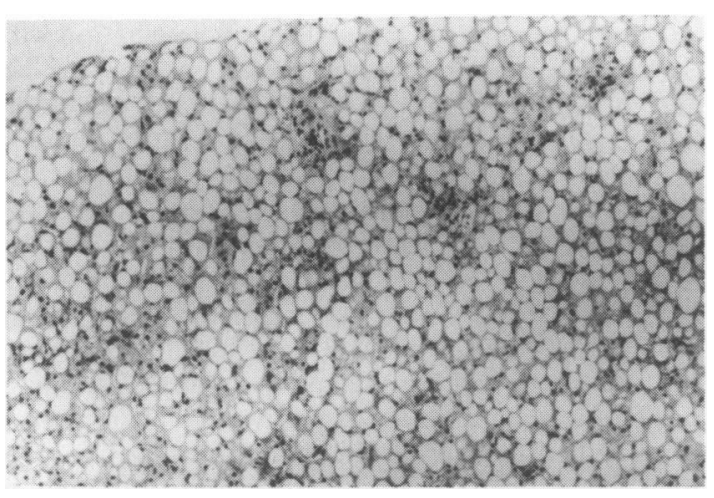

Figure 1 Liver biopsy from case 1 . Haematoxylin and eosin, magnification: $20 \times 6$.

weight, the liver was no longer palpable and liver function tests had returned to normal. A repeat small bowel biopsy after 9 months showed a marked improvement but there was still villous shortening.

\section{Case 2}

A 38 year old Caucasian male presented with a history of recent epigastric pain and weight loss of $10 \mathrm{~kg}$. He had a past history of obstructive jaundice assumed due to gall-stones at age 25 and a further laparotomy at age 29 for possible appendicitis complicated by postoperative jaundice. His alcohol intake was $320 \mathrm{~g} /$ week. No abnormality was noted on examination. Investigation revealed a normal full blood count and abnormal liver function tests - bilirubin normal, alanine transaminase (ALT) $79 \mathrm{IU} / 1$ (normal 5-50), ALP 113 IU/l (normal 25-90), gamma-glutamyl transferase $(\gamma \mathrm{GT}) 250 \mathrm{IU} / 1$ (normal $\leqslant 50)$. Gastroscopy, abdominal ultrasound, endoscopic retrograde cholangiopancreatography (ERCP) and isotope liver scan were all normal. Liver biopsy showed a slight to moderate lymphocytic and histiocytic infiltrate and occasional small foci of necrosis or inflammation and was reported as a mild non-specific hepatitis. The patient's condition continued to deteriorate with complaints of anorexia, depression and further weight loss. Examination now revealed $4 \mathrm{~cm}$ of smooth hepatomegaly. Liver function tests were more abnormal; bilirubin normal, ALT $77 \mathrm{IU} / 1$, ALP $176 \mathrm{IU} / 1$, $\gamma \mathrm{GT}$ $410 \mathrm{IU} / \mathrm{l}$. Repeat gastroscopy was normal but small bowel biopsy revealed subtotal villous atrophy and crypt hyperplasia. Repeat liver biopsy was unchanged.

On a gluten-free diet the patient gained $6 \mathrm{~kg}$ in weight and small bowel biopsy was normal a year later. Liver biopsy was not repeated but liver function tests remained abnormal.

\section{Case 3}

A 22 year old Caucasian woman presented with 8 months of generalized itching and $6 \mathrm{~kg}$ weight loss. Liver function tests had been found by her general practitioner to be abnormal prior to referral bilirubin normal, ALT 125 IU/1 (normal $\leqslant 37$ ), ALP $538 \mathrm{IU} / 1$ (normal $\leqslant 90$ ). She was on an oral contraceptive. On examination she was thin and excoriated. Further laboratory tests included smooth muscle antibody (titre 1/40) and reticulin antibody (titre 1/80), abdominal ultrasound revealed a normal parenchymal liver echo, liver biopsy showed changes only of a mild non-specific hepatitis.

Three months after presentation she described a self-limiting period of diarrhoea including nocturnal diarrhoea lasting for less than a month. Her itch had been relieved by cholestyramine but a mild iron deficient anaemia had appeared. ERCP showed a partial obstruction at the porta hepatis but no intrahepatic duct dilatation and was thought compatible with sclerosing cholangitis. Her liver function tests remained unchanged but $\gamma$ GT was noted to be disproportionately low $(51 \mathrm{IU} / \mathrm{l}$, normal $\leqslant 40)$ for the elevation of ALP, positive gliadin antibody was noted and a small bowel biopsy revealed almost total villous atrophy with increases in intra-epithelial lymphocytes, mitoses and crypt length.

After a year on a gluten-free diet her weight had increased by $4.5 \mathrm{~kg}$, her itch had gone and repeat small bowel biopsy was normal. However, further liver biopsy was unchanged and her liver function tests have remained abnormal.

\section{Discussion}

Although abnormalities of liver function are welldocumented in coeliac disease, ${ }^{1-4}$ liver damage is not generally recognized as a presenting feature sufficient to overshadow the underlying bowel disorder. Cooke and Holmes ${ }^{3}$ noted histological hepatic abnormalities in only 6 of over 400 patients with coeliac disease and only 12 out of 100 had elevated serum transaminases on more than one occasion. They did not feel that there was a striking association between coeliac disease and liver disease. In the first case the fatty liver and abnormal liver function tests appear to have been entirely secondary to coeliac disease; correction of the bowel abnormality produced normalization of the liver function tests and reversal of the hepatomegaly though it is unknown whether the histological appearances of the liver returned to normal. The histological appearances of the liver biopsy bear resemblances to those seen in alcoholic fatty liver and after jejunoileal bypass surgery suggesting that the damage may be due to nutritional changes or to 
exposure to hepatic toxins absorbed through damaged bowel wall. It would be reasonable to expect hepatic improvement after gluten withdrawal in such a case. Hagander et al. ${ }^{1}$ have described a retrospective survey of 74 adult coeliacs in a number of whom liver function test abnormalities (39\%) and histological abnormalities $(16 \%)$ were noted. In most of these cases the liver function tests improved on removal of gluten and in some of them, as in some of the cases reported by Pollock, ${ }^{2}$ there were histological findings similar to those in case 1 .

However, both of these papers also describe cases of chronic liver disease: chronic active hepatitis, cirrhosis and, in one case, hepatoma. The only discrete liver disease to be associated with coeliac disease is primary biliary cirrhosis, ${ }^{6-10}$ this is somewhat surprising as the liver disease associated with HLA B8, one of the HLA antigens associated with coeliac disease, is lupoid chronic active hepatitis rather than primary biliary

\section{References}

1. Hagander, B., Brandt, L., Sjoling, K., Berg, N.O., Norden, A. \& Stensom, M. Hepatic injury in adult coeliac disease. Lancet 1977, ii: 270-272.

2. Pollock, D.J. The liver in coeliac disease. Histopathology 1977, I: $421-430$.

3. Cooke, W.T. \& Holmes, G.K.T. Coeliac Disease. Churchill Livingstone, Edinburgh, 1984, pp. 231-232.

4. Green, P.A. \& Wallaeger, E.E. The clinical behaviour of sprue in the United States. Gastroenterology 1960, 38: 399-418.

5. Clinicopathological Conference. A case of malabsorption, intestinal atrophy and ulceration, cirrhosis and emphysema. Br Med J 1970, 3: 207-212.

6. Logan, R.F.A., Ferguson, A., Finlayson, N.D.C. \& Weir, D.G. Primary biliary cirrhosis and coeliac disease, an association? Lancet 1978, i: 230-233. cirrhosis. Primary biliary cirrhosis in association with coeliac disease is not ameliorated by a gluten-free diet and it would seem that they are two essentially independent conditions which sometimes share a common predisposition. Cases 2 and 3 fall more into this group, showing what appears to be a mild and non-specific chronic liver disease not directly related to gluten.

These cases illustrate yet another of the protean manifestations of coeliac disease: in all of them the hepatic abnormalities effectively obscured the diagnosis of coeliac disease for an average of 6 months. Coeliac disease deserves to be considered in all cases of unexplained hepatic dysfunction and, although in these cases the hepatic abnormalities were either reversible or relatively mild, it seems likely that prolonged hepatic damage of the sort seen in case 1 could, if untreated, become irreversible and lead to the more serious forms of liver disease already described.

7. Craxi, A., Puzello, G., Oliva, A. \& Pagliaro, L. Primary biliary cirrhosis and coeliac disease. Lancet 1978, i: 713-714.

8. Lee, F.I., Murray, S.M., Norfolk, D. \& Vasuder, K.S. Primary biliary cirrhosis and coeliac disease. Lancet 1978, i: 713 .

9. Iliffe, G.D. \& Owen, D.A. An association between primary biliary cirrhosis and jejunal villous atrophy? resembling coeliac disease. Dig Dis Sci 1979, 24: $802-806$.

10. Ollson, R., Kageri, I. \& Rydberg, L. On the occurrence of primary biliary cirrhosis and intestinal villous atrophy. Scand J Gastroenterol 1982, 17: 625-628. 